\title{
DEVELOPMENT OF AUTOMATED AERIAL PESTICIDE SPRAYER
}

\author{
P.D.P.R.Harsh Vardhan ${ }^{1}$, S.Dheepak ${ }^{2}$, P.T.Aditya ${ }^{3}$, Sanjivi Arul ${ }^{4}$ \\ ${ }^{1}$ Student, Department of Mechanical engineering, Amrita Vishwa Vidyapeetham, Tamil Nadu, India \\ ${ }^{2}$ Student, Department of Mechanical engineering, Amrita Vishwa Vidyapeetham, Tamil Nadu, India \\ ${ }^{3}$ Student, Department of Mechanical engineering, Amrita Vishwa Vidyapeetham, Tamil Nadu, India \\ ${ }^{4}$ Associate Professor, Department of Mechanical engineering, Amrita Vishwa Vidyapeetham, Tamil Nadu, India
}

\begin{abstract}
The World Health Organization estimates that there are 3 million cases of pesticide poison in each year and up to 220,000 deaths, primarily in developing countries. Organophosphates and carbonates, affect the nervous system. Others may irritate the skin or eyes. Some pesticides maybe carcinogens Others may affect the hormone or endocrine system in the body. Children, and indeed any young and developing organisms, are particularly vulnerable to the harmful effects of pesticides. Even very low levels of exposure during development may have adverse health effects. Pesticide exposure can cause a range of neurological health effects such as memory loss, loss of coordination, reduced speed of response to stimuli, reduced visual ability, altered or uncontrollable mood and general behavior, and reduced motor skills. An Automated Aerial Pesticide Sprayer is a basically a combination of a Blimp on a Quad copter frame. This project is to mainly overcome the ill-effects of pesticides on human beings(manual pesticide sprayers) and also to cover larger areas of fields while spraying pesticides in a short span of time when compared to a manual sprayer.
\end{abstract}

Keywords: pesticides, automated sprayer, blimp, quad copter.

\section{INTRODUCTION}

The World Health Organization estimates that there are 3 million cases of pesticide poison in each year and up to 220,000 deaths, primarily in developing countries. The potential health effects of pesticides include asthma, allergies, and hypersensitivity, and pesticide exposure is also linked with cancer, hormone disruption, and problems with reproduction and fetal development. Children are at greater risk from exposure to pesticides because of their small size: relative to their size, children eat, drink, and breathe more than adults. Their bodies and organs are growing rapidly, which also makes them more susceptible; in fact, children may be exposed to pesticides even while in the womb [1].

\subsection{Effects of Organophosphates and Pyrethroids:}

Acute organophosphate and carbonate exposure causes signs and symptoms of excess acetylcholine, such as increased salivation and perspiration, narrowing of the pupils, nausea, diarrhea, decrease in blood pressure, muscle weakness, and fatigue. Some organophosphates also have a delayed neurological reaction characterized by muscle weakness in the legs and arms. Among the most promising alternatives to organophosphates were synthetic pyrethroids. However, pyrethroids can cause hyper-excitation, aggressiveness, uncoordination, whole-body tremors, and seizures. Acute exposures in humans, usually resulting from skin exposure due to poor handling procedures, usually resolve within 24 hours. Pyrethroids can cause an allergic skin response, and some pyrethroids may cause cancer, reproductive or developmental effects, or endocrine system effects [2].In China, it's estimated that 500,000 people suffer pesticide poisoning annually, and some 500 of them die. Children seem to be greatly susceptible to the toxic effects of pesticides. The Natural Resource Defense Council has collected data which recorded higher incidence of childhood leukemia, brain cancer and birth defects. These results correlated with early exposure to pesticides.

\subsection{Effect on Mothers and Babies on Exposure to}

\section{Pesticides:}

Exposure to some pesticides can be 'hell' for a pregnant lady as it can easily lead to death of baby and even if the baby is produced will have lots of disorders or even loss of limbs.

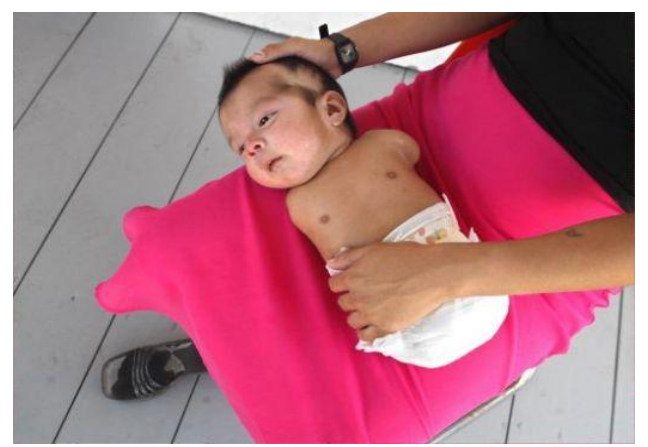

Fig.1 Harmful effects of pesticides 


\section{Methods Followed To Overcome the Problem}

There are various methods that are implemented today in various parts of the world to reduce the consequences caused to human beings (manual pesticide sprayers).

The ones in use today are:

1) Tractors (Deere) equipped with GPS system and huge sprayers which can automatically spray from the ground.

2) Using of a Remote Piloted Vehicle (RPV) for spraying pesticides (an RC helicopter). This method efficiently used the pesticides (10-15\% more efficient than manual spraying) and also could substitute 50 workers. Sadly this project was cancelled long ago.

3) Greenhouse pesticide spraying through pipelines and nozzle system.

4) Line following robot have been proposed to be an alternate.

\subsection{Drawbacks of these Solutions:}

1) Tractors occupy a large area to maneuver thus eating up large areas that could be used for plantations.

2) The Remote Piloted Vehicle uses a RC helicopter which can be hard to control and spraying can be not even.

3)The greenhouse method of spraying pesticides through pipelines running through supports are not suitable in open fields, which will demand high power pumps to pump pesticides over long distances due to major losses. Also it will be difficult to actually build a pipe system in a particular area of pest infestation and spray only there. (It occupies more time to locate the area of pest infestation and build a piping system as it involves a lot of labor and time too.)

4)Line following robots may be hard to implement in an actual scenario as they are not good in rough and hilly terrains. Carrying the equipment and the pesticide can bring much complexity to maneuvering and controlling.

\section{PROPOSED SOLUTION}

\subsection{Aerial Automated Pesticide Sprayer:}

AAPS is basically a blimp (zeppelin) integrated into a quad copter to spray pesticides in open fields. The main aim of this system is to reduce the ill-effects to humans, lessen the time used for spraying, use economically the pesticides and to use under any climatic conditions.

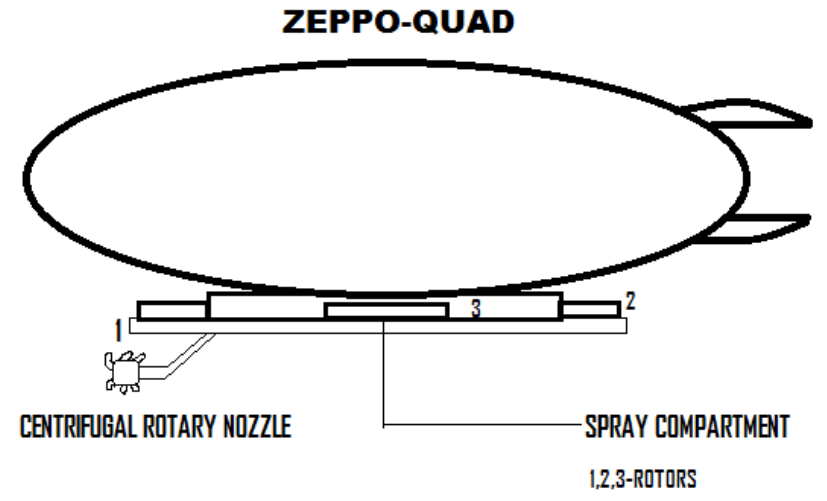

Fig2. Schematic Of The Proposed Idea

A Quad copter with a mechanism which can come back to its reference or initial position is used here. This increases the control of the Zeppo-quad rotor. The blimp gives the lift and the propellers take care of the steering part and the quad copter takes care of the control and also supports the lift and propelling of the Zeppo-quad rotor. The balloon is connected to the quad copter (mainly) so that the gondola (spray platform) can be lowered, to ease spraying which is explained later.

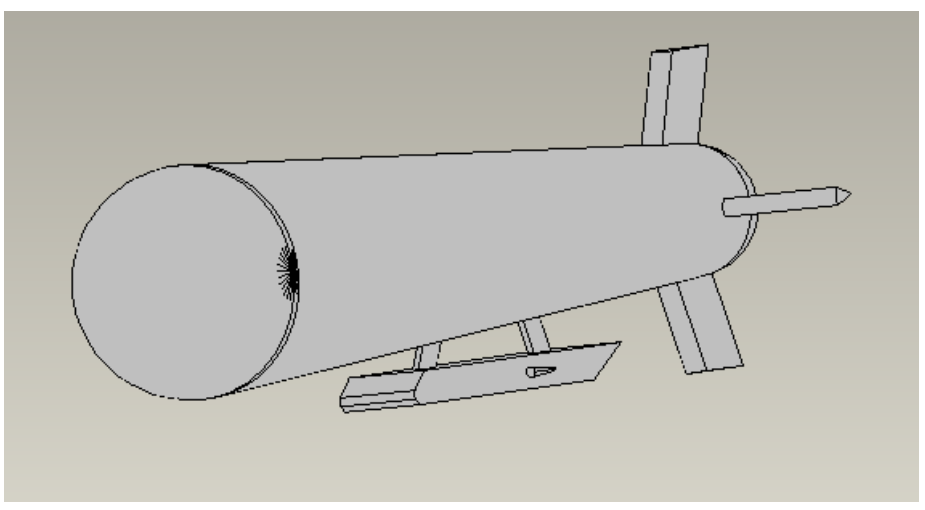

Fig 3 CAD model of a Blimp

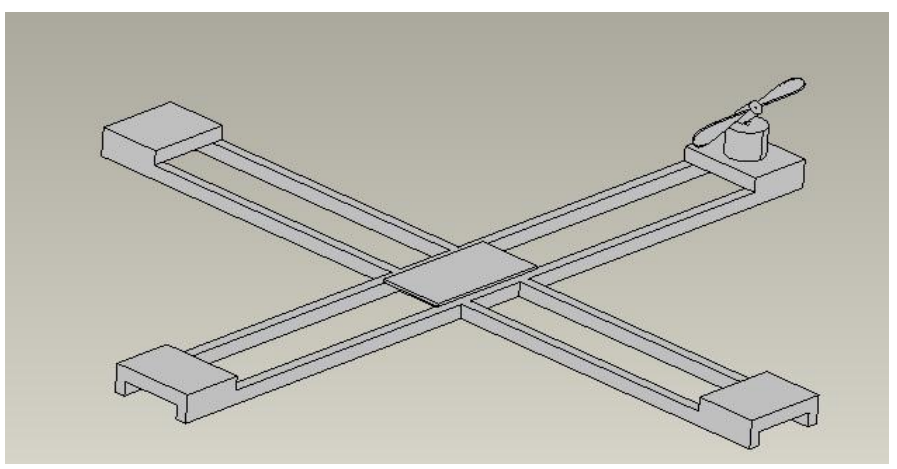

Fig 4 CAD model of a Quad frame with one rotor 


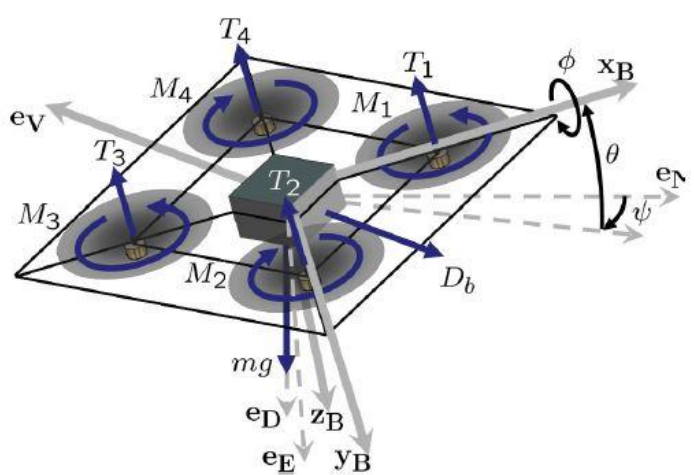

Fig 5 Free Body Diagram of a Quad copter [4]

There are three methods of implementing this:

1) A GPS system is integrated into it. In areas where multiple cropping patterns are implemented, certain cropping patterns, or certain pest infested area alone can be targeted and commanded to be quarantined. Eg.Paddy - brown plant hopper, which produces brown circular patches of infested crops in a paddy plantation.

2) A proximity sensor can be attached in the front face of the blimp which takes a turn on encountering a sign board and thus follows a trajectory which covers the whole field area.

3) A Zeppo-quad rotor can also be used to spray liquid fertilizers over a uni-crop patterned field by just giving the length and breadth of the field and also specifying a reference co-ordinate.

\subsection{Advantages}

Can be used to spray on hilly terrains Eg. On tea plantations as it's aerially sprayed and the terrain is of no consequence. Can be used to spray uni-crop, multi-crop pattern and almost any cropping pattern in fields. Increases the efficiency of spraying (like we saw in the RPV) and also can substitute 50 workers thus saving 50 workers from the harmful effects. Reduces the time for spraying when compared to manual spraying. Pesticides are evenly sprayed. More area can be brought under cultivation (unlike the GPS integrated Deere tractors based automation which needs some area for them to maneuver on the ground). Increases the turnover from cultivation, by using AAPS, Thus it increases the economy of the country as a whole. Another advantage is that the spray platform (the gondola of the blimp) can be lowered to any desired level which aids the spraying of pesticide from beneath the leaves. Also it can be used to spray from the top as well and thus covering a large verity of crops for which AAPS can be used. Various attachments like multipoint nozzle rotors and probes with multiple nozzles fitted to it can also be used to increase its usage. The pesticides can be mixed and carried in containers (cartridges) which can be pressurized to spray by a piston arrangement (similar to a syringe).

\section{WORKING OF ZEPPO-QUAD ROTOR}

The piston which pushes out the pesticide in the cartridge will be actuated by a cam shaped like shown in the picture. When the cam completes one rotation the piston will be in its max displacement from the home position and it comes back to its home position using a spring. The rotation of the cam is brought about by the worm and worm wheel arrangement driven by a small dc motor. So in one rotation of the cam the whole cartridge is used and the gear ratio should be correspondingly adjusted. A sensor senses when the cam has completed its one rotation (piston reaches the maximum displacement from home position) and stops the zeppo-quad rotor which will then continue moving when the cartridge is replaced. The cartridge can be replaced when the piston has come back to its home position only. An alternate way of actuating the piston is to connect the piston to a connecting rod connected from a disc, which is driven by a worm and worm wheel mechanism which in turn is run by a DC motor. The same sensors can be used here to sense the position of the piston, thus ensuring all the pesticide has been removed. The cartridge can be removed only when the piston comes back to its home position (the piston would hinder the removal of the cartridge if it was inside it).

\section{SOME IMPORTANT CONSIDERATIONS WHILE DESIGNING A QUAD}

\subsection{Frame Characteristics}

The most suitable way is to begin with is the skeleton of the Quad copter, the frame. The frame is considered the largest (in volume) component used in the Quad copter and a very important one. Choosing a frame to fulfill the required need is essential, critical, and also not simple. The frame should be light-weight yet strong to tolerate possible accidents and crashes, it has also to be thin [5].

\subsection{Propeller Characteristics}

The mechanical lifting element of the Quad copter is the propellers. High quality propellers are made by different manufacturers to deliver great performance of lifting power at a very small weight; to minimize the torque needed for spinning them by the motor's rotor. As mentioned propellers are manufactured from a variety of materials such as; plastic, which is cheap, available, and light, but is by nature fragile meaning it could be damaged or broken easily. Wood is tough and mid-priced but is certainly heavier than plastic. Lastly is the carbon fiber which is tough and light-weight but more expensive than the previous two types. 


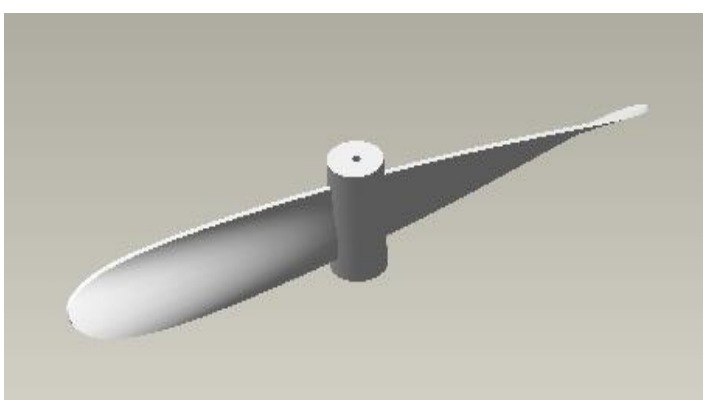

Fig 6.CAD model of a Quad copter Propeller

Choosing a suitable size is quite crucial, since it affects the power consumption, the overall weight, and how sturdy and reliable (stable-wise) the Quad copter is. Increasing the size of the propellers means higher thrust delivered, but that comes at the cost of more torque required to spin them by the motors. Having that in mind, the criterion for choosing the propellers is mainly dependent on the model that is being built and its overall weight. Importantly is the fact that four propellers are needed, but with a pair being oriented for clockwise (CW) direction and the other pair for the counter-clockwise (CCW) direction.

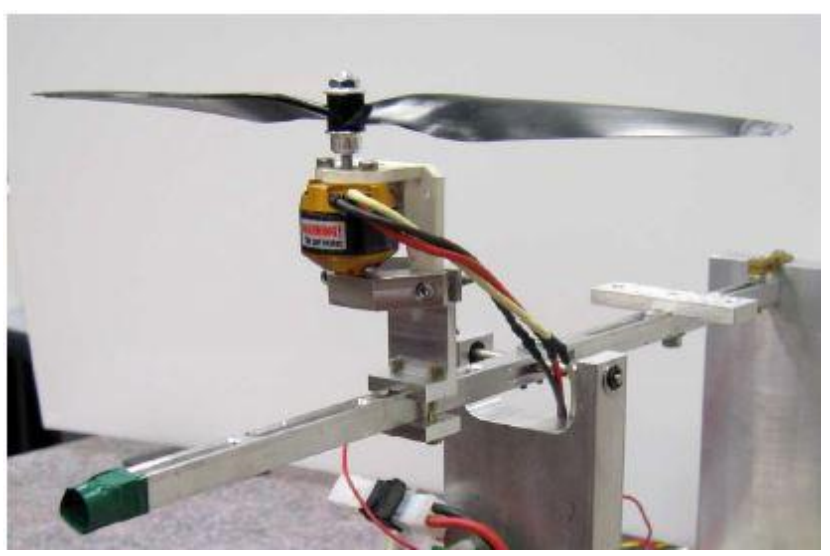

Fig 7.Testing setup of Propeller

\subsection{Electronic Speed Controller:}

ECS's are required to run the BLDC motors in the Quad copter. The ESC is a standalone chip that's connected to the receiver's control channels and then coupled with the BLDC motor. For a better understanding of the ESC, it's more conventional to consider it as a pulse-width modulation (PWM) controller for the BLDC motors. PWM in short is a great way of controlling some modern electronics such as a BLDC motor, a fast variation between the motor being fully off and fully on powered, more conveniently described as a percentage called the duty cycle. Controlling the duty cycle means controlling the speed of the BLDC motor without any losses and also without affecting the load [6].

\subsection{Software \& Control Design:}

After selecting the right hardware components, the next step is to have a proper design for the code that will run on the Quad copter. This phase is the hardest of all and needs so much patience and constant tuning and monitoring. Having a snappy control loop is essential as the Quad copter tries to selfstabilize itself, without such a loop, the flight could be sluggish with a huge amount of errors, which will be also accumulative and eventually would result in a crash with unexpected consequences. Starting off with a simple, yet much enriched flow chart of how should the program embedded in the micro-controller look like and behaves. A flow chart is very similar to the block diagram; brief and comprehensive [6].

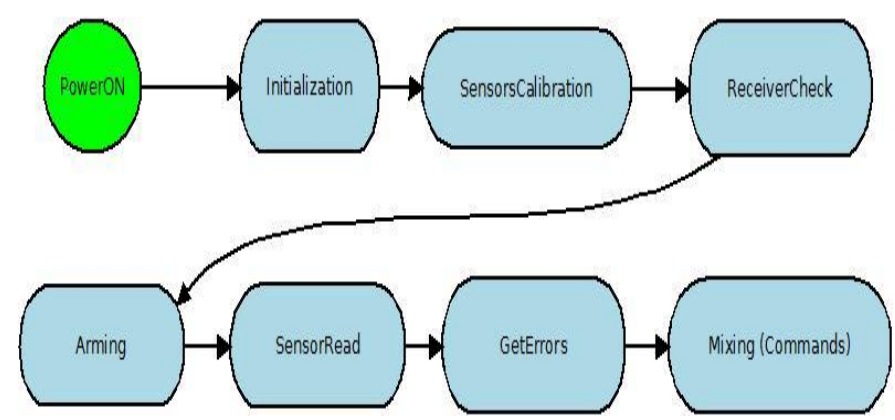

Fig 8 Schematic flow chart showing the initial setup and calibration steps from startup [5].

The process begins with providing the power through the battery, at this instance hardware components will initialize and boot. After that the sensors will be calibrated, taking the nominal value that they "see" and consider it as the reference. The receiver would then start testing the frequency and channels in order to make sure that it is correctly connected with the transmitter. The phase called arming can now take place, setting different modes and configuration such as; turning the whole system off (disarm). The next two steps are mainly getting the values that the sensors read and then sending those values to the proportional-Integral (PD) which will get the error and output the final values with the help of the desired values from the transmitter and receiver. To conclude, mixing is the process of sending the commands to the motors based on the desired input plus the output value generated from the PD.

\subsection{Algorithm of functioning of AAPS:}

1) Using a pre-programmed chip: assume that breadth of field sprayed in one straight run of blimp be $30 \mathrm{~cm}$.

Step 1: start

Step 2: Get values of length and breadth of field

Step 3: Trigger the motion of the piston towards the extreme end of the cartridge as the blimp starts moving

Step 4: If - piston at extreme end of the cartridge, go to step 8 , else go to step 5 
Step 5: Move the blimp till *present position < breadth* and if $*$ present position $<$ breadth $=$ move*; else go to step 6

Step 6: Turn 90 degrees left move $30 \mathrm{~cm}$, store this value in L1 and then turn again 90 degrees left

Step 7: Move forward

Step 8: Stop

2) Using a proximity sensor: using previous assumption

Step 1: Start

Step 2: Trigger the motion of the piston to the extreme end of the cartridge

Step 3: Check if signal is received from sensor (that piston has reached the extreme end)

Step 4: If yes, go to Step 12 else, go to step 5

Step 5: Move forward

Step 6: Check if signal is detected from the proximity sensor at the front end of blimp.

Step 7: If yes, go to Step 8 else, go to step 5

Step 8: Turn 90degrees to the left, move $30 \mathrm{~cm}$, again turn 90degrees left and move forward

Step 9: Check if signal is detected from next proximity sensor Step 10: If yes, go to Step 11 else, go to step 5

Step 11: Turn 90degrees right, move $30 \mathrm{~cm}$ forward turn 90 degrees right move forward

Step 12: Stop

\section{BLIMP DESIGN}

It has no rigid internal structure and obtains the shape of its hull only by internal overpressure. The only solid parts are the gondola and the tail fins. The advantages of this non-rigid structure are obvious. Not only is the ship many times lighter than a comparable rigid airship, but also almost as resistant to weather conditions, if an adequate envelope material is chosen.

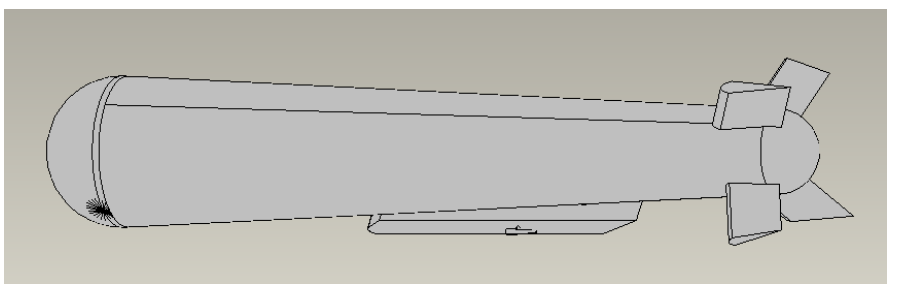

Fig 9.Blimp model

\subsection{Considerable Advantages of a Blimp:}

1) A minimum of expenses has been realized to achieve the aims of the project.

2) The airship may be de- and reassembled for transportation purposes in very short time. The most economical of these forces from the production of lift point of view is undoubtedly the static lift wherein buoyant force is generated by the displacement of a portion of the supporting medium by the body and for airborne vehicles (like Blimps), it is the balloon.

\subsection{Various Lift Forces in a Blimp:}

The inefficiency of the static lift vehicle comes when it is required to move through the surrounding medium. Due to the nature of displacement buoyancy, these vehicles tend to be very large and, as a result, they develop a great deal of dynamic drag when in motion. The dynamic effects of the motion can be used to an advantage, however, if the motion can be used to generate lift. By shaping the body, or a portion thereof, as a lift producing foil, a lifting force may be developed to support the weight of the body, provided sufficient forward speed is attained. A principle disadvantage of the dynamic lift vehicle is that it requires forward motion of some finite velocity to generate the lift. As a result, this vehicle can neither fly very slowly nor can it remain airborne at zero forward velocity (hover). If these attributes are required, one must provide some sort of internal powering for the static lift, such as a vertical jet exhaust, or a propeller with a vertical down flow [3].

Having defined these primary sources of lifting force, one might observe that it is possible to use two of these sources, or even all three, in combination. By doing so, one moves from the pure lifting force source, for example static lift, to a hybrid source, such as a partial static lift and a partial dynamic lift. This is exactly the technique used here. Its envelope produces static lift, while the two motors provide powered static lift and is also supported by the quad copters, and dynamic lift when the hull is positioned in a way shown.
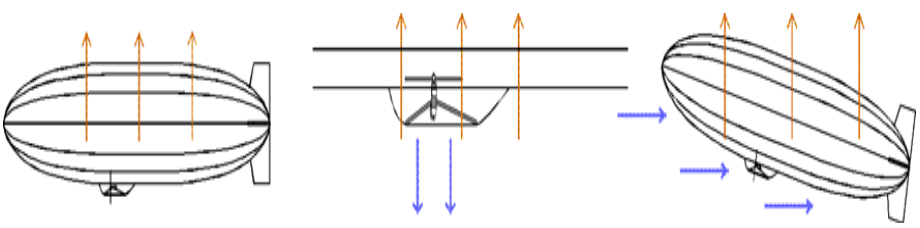

Fig 10.Showing Static, Powered Static, and Dynamic Lift

\section{THE ULTIMATE OBJECTIVE}

This method can be used in all situations. Of used for highly toxic pesticides this will not lead to unemployment problems. This can also be used in places where laborers are hard to find. They can hasten the pesticide spraying process thereby lowering the causalities due to pesticide exposure, preventing encounters with venomous snakes such as Russell's viper and cobra which can be found regularly in fields thereby reducing the amount of snake bite cases. Also keeps the food chain healthy. Since they are sprayed from lower altitude, environmental pollution can be reduced.

\section{CONCLUSIONS}

This method can be used in all situations. Of used for highly toxic pesticides this will not lead to unemployment problems. This can also be used in places where labourers are hard to find. They can hasten the pesticide spraying process thereby 
lowering the causalities due to pesticide exposure, preventing encounters with venomous snakes such as Russell's viper and cobra which can be found regularly in fields thereby reducing the amount of snake bite cases. Also keeps the food chain healthy. Since they are sprayed from lower altitude, environmental pollution can be reduced.

\section{REFERENCES}

[1] International code of conduct on the distribution and use of pesticides by Food and Agricultural Organization of the United Nations Rome 2001.

[2] Environmental health practitioner manual.

[3] Ruegg, Lutz and Ruegg, "The Airship 'Simon'Building and construction of a remote controlled blimp" TataMcGrawHill, London. Ed. 4 Vol.2 pp. 201-212.

[4] Gabriel M. Hoffmann, Haomiao Huang Steven L. Waslander Claire J. Tomlin "Quad rotor Helicopter Flight Dynamics and Control: Theory and Experiment".

[5] AbdallahZaid Al Kilani, SaifallahQasimSearch-AndRescue Remote Sensing Quad rotor UAV, GermanJordanian University, Project Entry Documentation For National Technology Parade 2010.

[6] David Malgoza, Engers F DavanceMercedes ,Stephen Smith Joshua West "Quad-Copter:Autonomous Surveillance Robot, Senior Design I Documentation under Dr. Samuel Richie ,Summer-Fall 2010.

[7] Pesticides - Harmful effects and emergency response, Extracted from http://pest.ca.uky.edu/EXT/ukgh/Exposure.pdf.

[8] Potential health effects of pesticides, Available : http://pubs.cas.psu.edu/freepubs/pdfs/uo198.pdf.

[9] Effects of Pesticides, Available:http://www.globalhealingcenter.com/effectsof-pesticides/effects-of-pesticides.

[10] http://en.wikipedia.org/wiki/Pesticide.

[11] http://www.epa.gov/opp00001/health/human.htm\#healt heffects.

[12] http://toxipedia.org/display/toxipedia/Effects+of+Pestic ides+on+Human+Health.

[13] http://www.nkkhoo.com/2013/03/23/automation-inagriculture-like-spraying-pesticide-and-fertilizer.

[14] http://www.bvsde.paho.org/bvsacd/cd37/1176.pdf.

[15] Stepniewski, W. Z., Rotary-wing aerodynamics, Dover Publications, New York, NY, 1984.

[16] Newman, S., The Foundations of Helicopter Flight, Halsted Press, New York, NY, 1994.

[17] Altug, E., Ostrowski, J. P., and Taylor, C. J., "Quadrotor Control Using Dual Camera Visual Feedback," In Proceedings of the IEEE International Conference on Robotics and Automation, Taipei, Taiwan, Sept 2003, pp. 4294-4299.

[18] "DraganFly-Innovations DraganFlyer IV," 2006, http://www.rctoys.com.
[19] Hoffmann, G., Rajnarayan, D. G., Waslander, S. L., Dostal, D., Jang, J. S., and Tomlin, C. J., "The Stanford Test bed of Autonomous Rotorcraft for Multi Agent Control (STARMAC)," In Proceedings of the 23rd Digital Avionics Systems Conference, Salt Lake City, UT, November 2004.

[20] Escare no, J., Salazar-Cruz, S., and Lozano, R., "Embedded control of a four-rotor UAV," Proceedings of the AACC,American Control Conference, Minneapolis, MN, June 2006, pp. 3936-3941.

[21] Nice, E. B., Design of a Four Rotor Hovering Vehicle, Master's thesis, Cornell University, 2004.

[22] Pounds, P., Mahony, R., and Corke, P., "Modelling and Control of a Quad-Rotor Robot," In Proceedings of the Australasian Conference on Robotics and Automation, 2006.

[23] Mokhtari, A. and Benallegue, A., "Dynamic Feedback Controller of Euler Angles and Wind parameters estimation for a Quadrotor Unmanned Aerial Vehicle," In Proceedings of the IEEE International Conference on Robotics and Automation, New Orleans, LA, April 2004, pp. 2359-2366.

[24] Pounds, P., Mahony, R., Gresham, J., Corke, P., and Roberts, J., "Towards Dynamically-Favourable QuadRotor Aerial Robots," In Proceedings of the Australasian Conference on Robotics and Automation, Canberra, Australia, 2004.

\section{BIOGRAPHIES}

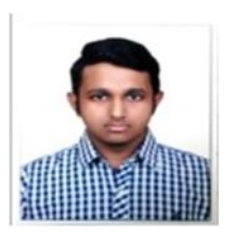

P.D.P.R.HARSH VARDHAN, Student , Amrita university, phv27m@gmail.com

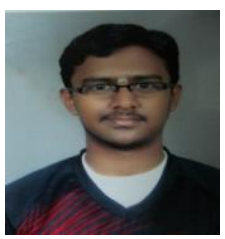

S.DHEEPAK, Student , Amrita university, dheepakxs3@gmail.com

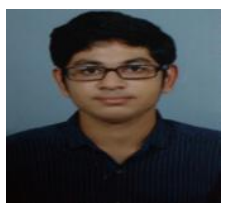

P.T.ADITYA, Student , Amrita university, puttaparthy.aditya@gmail.com

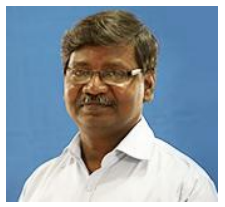

Dr. SANJIVI ARUL, Associate Professor, Amrita university, s_arul@cb.amrita.edu 